\title{
Generalized Lymphadenapathy - An Unusual Presentation of Sarcoidosis
}

APARNA DAS, ${ }^{1}$ MARUF H CHOWDHURY, ${ }^{2}$ ABMG MOSTAFA,${ }^{3}$ M HASAN, ${ }^{3}$ MA KAHHAR ${ }^{4}$

\begin{abstract}
:
Sarcoidosis is a systemic granulomatous disease of unknown origin. It is manifested pathologically by the presence of noncaseating granulomas containing multinucleated giant cells, although other granulomatous diseases may produce identical findings. The vast majority of patients have thoracic sarcoidosis with bilateral hilar lymphadenopathy andl or bronchonodular pulmonary infiltrates on chest radiographs. About $40 \%$ of patients have extrathoracic disease involving the eye, skin, lymph nodes, liver, spleen, heart, and musculoskeletal or nervous system. Here we report a case of sarcoidosis who presented only with generalized lymphadenopathy.
\end{abstract}

Keywords: Sarcoidosis, Lymphadenopathy

\section{Introduction:}

Sarcoidosis is a multisystem granulomatous disorder characterized pathologically by the presence of noncaseating granulomas in involved organs, occurring in patients between 10 and 40 years of age in 70 to 90 percent of cases. It typically presents with bilateral hilar lymphadenopathy, pulmonary reticular opacities, and skin, joint or eye lesions. Lung is the most common organ involved, which is $95 \%$ followed by skin and lymph nodes, $15.9 \%$ and $15.2 \%$ respectively. ${ }^{1,2}$ Extrapulmonary manifestations vary on the basis of sex, age at presentation, and ethnicity. Women are more likely to have neurologic or ocular involvement, while men more frequently have abnormalities of calcium homeostasis. Up to 30 percent of patients present with extrapulmonary disease ${ }^{1}$.The incidence of the disease varies among geographical regions. It occurs more commonly, three to four times in blacks in the United States of America. ${ }^{3}$ Sarcoidosis, though rare in our community, should still be considered in the differential diagnosis of patients with the typical presentation after excluding tuberculosis. Here we report a case of sarcoidosis for its unusual presentation without typical clinical features.

1. Assistant Professor. Department of Medicine, Dhaka Medical College\& Hospital

2. Registrar, Department of Medicine, Dhaka Medical College \&Hospital

3. Medical Officer. Department of Medicine, Dhaka Medical College \&Hospital

4. Professor, Department of Medicine, Dhaka Medical College \&Hospital

Correspondence: Dr. Aparna Das, Assistant Professor, Department of Medicine, Department of Medicine, Dhaka Medical College \& Hospital. Mobile: 01914978719

\section{Case Report:}

A 32 year, Muslim, male, businessman from Kishorganj was admitted into DMCHwith the complaints of progressive generalized lymphadenopathy for 6 months. He had no history of fever, cough, respiratory distress, chest pain, weight loss, night sweat, joint pain, rash and no contact with TB patient. His bowel and bladder habit was normal and he was smoker,normotensive andnon-diabetic. On examination, patient was anxious, average body built and not anaemic. He had generalized lymphadenopathy involving inguinal, axillary and cervical LN.They were mobile, non tender, firm in consistency, free from underlying structure and overlying skin. Largest one is in inguinal region; size

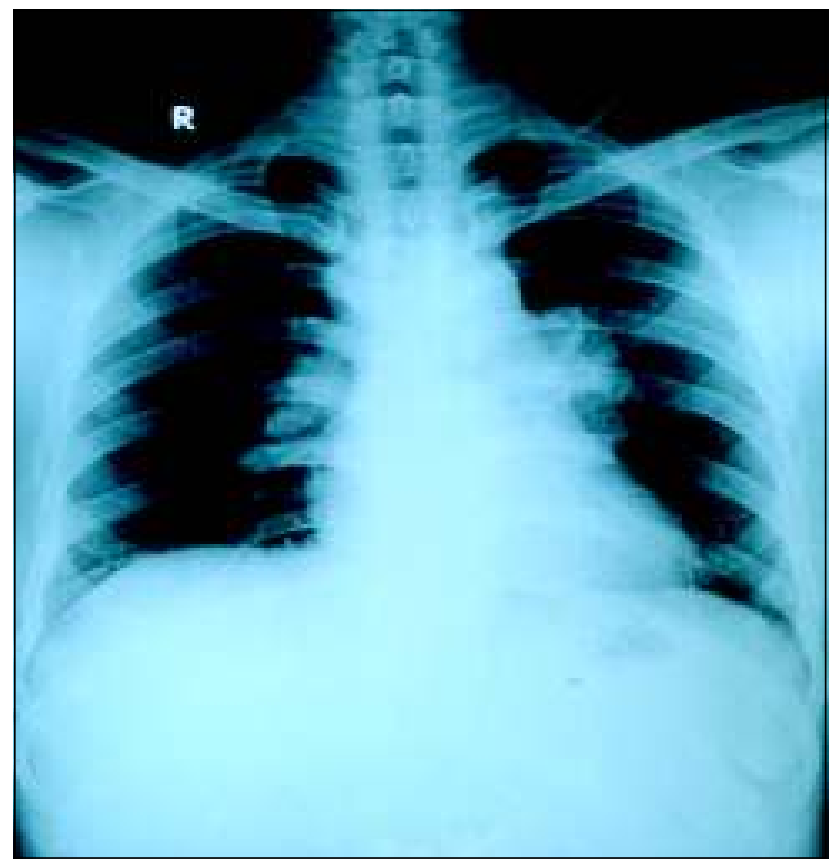

Fig-1: $C X R \quad P / A$ view showing bilateral hilar lymphadenopathy 
was 3 X $3 \mathrm{~cm}$. His pulse was $85 \mathrm{~b} / \mathrm{min}$, BP was $120 / 75 \mathrm{mmHg}$ and other systems revealed no abnormality. $\mathrm{His} \mathrm{Hb}$ was $14.20 \mathrm{~g} / \mathrm{dl}$, ESR $45 \mathrm{~mm} 1^{\text {st }}$ hour, WBC 6000/cmm, N 64\%, L $31 \%$, E 03\%, M 02\%, PBF Shows RBC - Normocytic and normochromic, WBCs - mature with normal count, Platelate were adequate. Sputum for AFB showed negative for AFB, Tuberculin test -negative, CXR- Bilateral hilar lymphadenopathy, USG of W/A - Para aortic, inguinal and femoral Lymphadenopathy, ANA - Negative, RA- negative, S. Alkaline Phosphatase - $204 \mathrm{U} / \mathrm{L}$, Calcium - $10.25 \mathrm{mg} /$ dl,Albumin- $4.59 \mathrm{gm} / \mathrm{dl}, 24$ hours urinary calcium- $539 \mathrm{mg} / 24$ hours (reference value 100 - 300), CT scan of chest Suggestive of Extensive mediastina Lymphadenopathy and Bilateral Hilar Lymphadenopathy. Bronchoscopy and BAL for cytology revealed normal findings. Histopathology report of inguinal lymph node- Non caseating granulomatous lymphadenitis, histologically compatible with sarcoidosis.

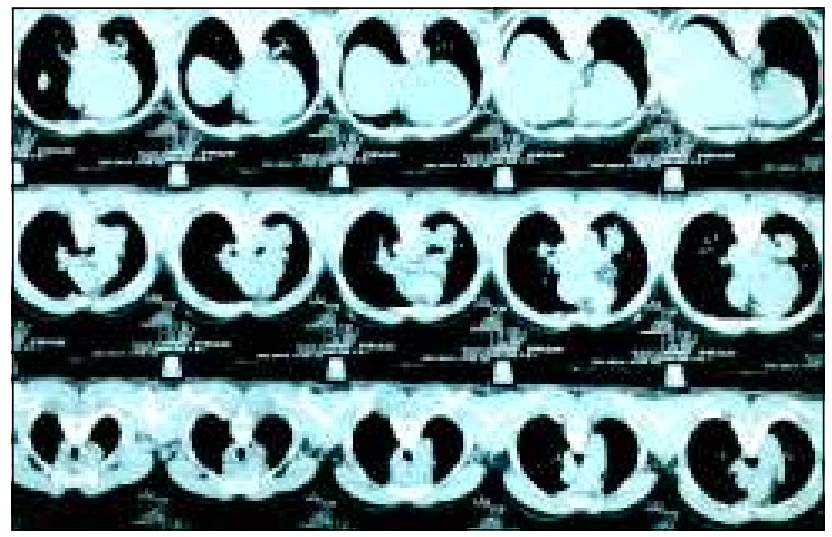

Fig 2 : CT scan of chest - Suggestive of Extensive mediastinal Lymphadenopathy and Bilateral Hilar Lymphadenopathy

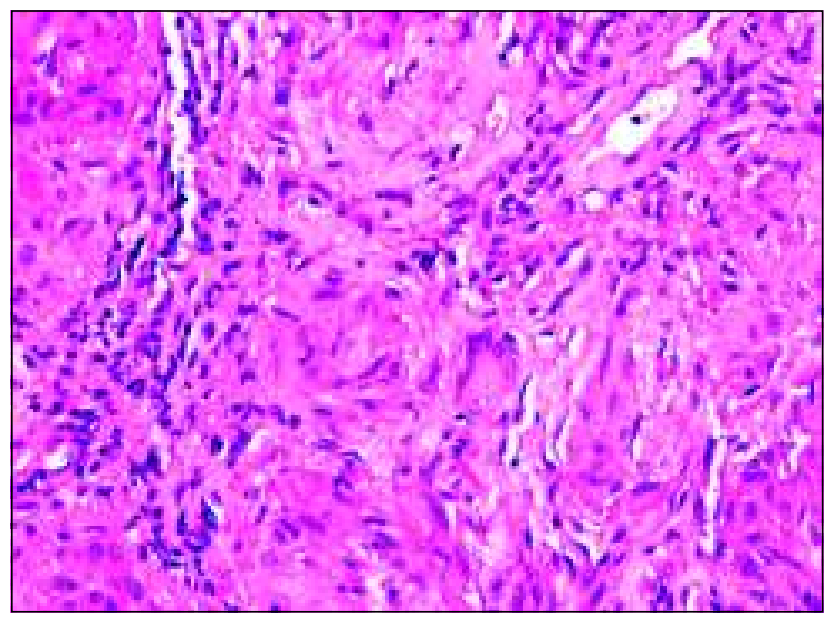

Fig.-3: Histopathology report of inguinal lymph node-Non caseating granulomatous lymphadenitis, histologically compatible with sarcoidosis.
ACE level was $101 \mathrm{U} / \mathrm{L}$ ( Ref: 8-65U/L) Though we cannot do the CD4 and CD8 level of BAL, diagnosis of sarcoidosis was reached following excisional biopsy of a superûcial lymph node, following negative bronchoscopy and unremarkable haematological, biochemical and serological work-up.

\section{Discussion :}

Sarcoidosis can involve all organ systems, which the most prominent sites of extrapulmonary disease include the skin, eyes, reticuloendothelial system, musculoskeletal system, exocrine glands, heart, kidney, and central nervous system.This case presented with generalized lymphadenopathy and vague symptoms of fatigue, weakness. The presentation that favors sarcoidosis was bilateral hilar lymphadenopathy in chest X-ray. The stage of pulmonary involvement is based on the chest radiograph. Stage I is defined by the presence of bilateral hilar lymphadenopathy, often accompanied by right paratracheal node enlargement. Fifty percent of affected patients exhibit bilateral hilar lymphadenopathy as the first manifestation of sarcoidosis. Regression of hilar nodes within one to three years occurs in 75 percent of such patients, while 10 percent develop chronic enlargement that can persist for ten years or more. Stage II consists of bilateral hilar lymphadenopathy and reticular opacities. Reticular opacities occur predominantly in the upper lobe. Patients with stage II disease usually have mild to moderate symptoms, most commonly cough, dyspnea, fever, and/or easy fatigue. Stage III consists of reticular opacities with shrinking hilar nodes. Stage IV disease is characterized by reticular opacities with evidence of volume loss, predominantly distributed in the upper lung zones. Marked traction bronchiectasis, extensive calcification and cavitation, or cyst formation may be seen. From the above staging, this patient presented with Stage II sarcoidosis. Chest CT can demonstrate a variety of abnormalities in patients with sarcoidosis. ${ }^{5,6}$ Hilar and mediastinal lymphadenopathy, beaded or irregular thickening of the bronchovascular bundles, nodules along bronchi, vessels and subpleural regions, bronchial wall thickening, ground glass opacification, parenchymal masses or consolidation, parenchymal bands, cysts, traction bronchiectasis, and fibrosis with distortion of the lung architecture.

Cutaneous involvement is seen in up to 20 percent of patients with sarcoidosis. ${ }^{4} \mathrm{~A} \mathrm{~m}$ aculopapular eruption is the most common subacute lesion. It usually involves the alae nares, lips, eyelids, forehead, rear of neck at the hairline, and/or previous trauma sites (for example, scars and tattoos). Waxy, pink nodular lesions are frequently distributed on the face, 
trunk, and extensor surface of the arms and legs. Plaque-like lesions can occur in chronic sarcoidosis including lupus pernio, a violaceous discoloration of the nose, cheeks, chin, and ears. Erythema nodosum (EN) is a component of Lofgren's syndrome and associated with a good prognosis and spontaneous remission. ${ }^{5}$ Atyical lesions may be ulcerative, psoriasiform, hypo pigmented, follicular, angiolupoid, rosacea-like, or morpheaform. ${ }^{6}$ In this patient, no skin lesion was found. No evidence of keratoconjunctivitis sicca or anterior and posterior uveitis and salivary gland was found.

Reticuloendothelial system disease is common in sarcoidosis, manifest as peripheral lymphadenopathy (40 percent), hepatomegaly (20 percent), non-caseating granulomas on liver biopsy with or without hepatomegaly ( 75 percent), and splenic enlargement (25 percent). Hypersplenism can lead to anemia, leukopenia, and thrombocytopenia. Our patient had features of only generalized lymphadenopathy, no other features of haematological abnormality.

Calcium metabolism abnormalities are the most common renal and electrolyte abnormalities observed among patients with sarcoidosis. The defect in calcium metabolism is due to extrarenal production of calcitriol by activated macrophages. Patients may present with hypercalciuria (occurs in up to 50 percent of cases), hypercalcemia (which occurs in 10 to 20 percent), and nephrocalcinosis. If untreated, renal calcium deposition can lead to chronic renal failure and end-stage renal disease. The calcium level was upper limit of normal in this patient however urinary calcium was high. The erythrocyte sedimentation rate (ESR) is frequently elevated and a positive rheumatoid factor can exist. In our patient also having high ESR, negative tuberculin test and high serum angiotensin converting enzyme (ACE) level which was in favour of sarcoidosis. ACE level is elevated in 75 percent of untreated patients with sarcoidosis. ${ }^{7}$ False positive results occur in less than $5 \%$ of cases. Bronchoalveolar lavage (BAL) can be used as an adjunctive measure to support the diagnosis of sarcoidosis by demonstrating a reduced number of CD8 cells, an elevated CD4 to CD8 ratio, and an increased amount of activated T cells, CD4 cells, immunoglobulins, and IgG-secreting cells. ${ }^{8,9}$ BAL can also be helpful in excluding infections as an alternative diagnosis. A CD4 to CD8 ratio less than one had a 100 percent negative predictive value for sarcoidosis. The triad of a CD4 to CD8 ratio greater than four to one, a lymphocyte percentage greater than or equal to 16 percent, and a transbronchial biopsy demonstrating non-caseating granulomas were the most specific tests for sarcoidosis.

The characteristic morphologic feature of sarcoidosis is the non-caseating granuloma. The granuloma is a focal, chronic inflammatory reaction consists of epithelial cells, monocytes, lymphocytes, macrophages, and fibroblasts. Multinucleated giant cells are frequently found among the epithelioid cells within the granuloma follicle and often have cytoplasmic inclusions, such as asteroid bodies, Schaumann bodies, and birefringent crystalline particles (calcium oxalate and other calcium salts). ${ }^{10}$ Most sarcoid granulomas gradually resolve and leave few or no residual manifestations of previous inflammation. However, haematological and other investigations do not suggestive of tuberculosis, lymphoma and leukaemia. The final diagnosis of sarcoidosis was based on the histopathological excision biopsy of the inguinal lymph node.

\section{Conclusion:}

The diagnosis of sarcoidosis requires compatible clinical and radiographic manifestations, exclusion of other diseases that may present similarly and histopathology detection of noncaseating granulomas. Biopsies should be performed on accessible lesions, example from palpable lymph nodes, subcutaneous nodules, cutaneous lesions, an enlarged parotid gland, or an enlarged lacrimal gland. Fiberoptic bronchoscopy with transbronchial biopsy should be performed if an accessible peripheral lesion cannot be identified.

\section{Conflict of Interest : None}

\section{References:}

1. Baughman RP, Teirstein AS, Judson MA, Rossman MD, Yeager H, Jr., BresnitzEA, DePalo L, et al. Clinical characteristics of patients in a case control study of sarcoidosis. Am J RespirCrit Care Med 2001;164(10 Pt 1): 1885-1889.

2. Rizzato G, Tinelli C. Unusual presentation of sarcoidosis. Respiration 2005;72(1):3-6.

3. Thomas KW, HunninghakeGW. Sarcoidosis. JAMA 2003; 289(24): 3300-3303.

4. Roberts SD, MirowskiGW, Wilkes D, KwoPY, Knox KS. Sarcoidosis. Part II: extrapulmonary and systemic manifestations. J Am AcadDermatol 2004;51(4):628-630.

5. Neville E, Walker AN, James DG. Prognostic factors predicting the outcome of sarcoidosis: an analysis of 818 patients. Q J Med 1983;52(208):525-533.

6. English JC, 3rd, Patel PJ, Greer KE. Sarcoidosis. J Am AcadDermatol 2001;44(5):725-743.

7. Studdy PR, Bird R. Serum angiotensin converting enzyme in sarcoidosis - its value in present clinical practice. Ann ClinBiochem 1989; 26 (Pt 1)(13-18).

8. Winterbauer RH, Lammert J, Selland M, Wu R, Corley D, Springmeyer SC. Bronchoalveolar lavage cell populations in the diagnosis of sarcoidosis. Chest 1993;104(2):352-361.

9. Perez RL, Kimani AP, King TE, Jr., Aguayo SM, Roman J. Bronchoalveolar lavage fluid D dimer levels are higher and more prevalent in black patients with pulmonary sarcoidosis. Respiration 2007;74(3):297-303.

10. Myers JL, Tazelaar HD. Challenges in pulmonary fibrosis: 6-Problematic granulomatous lung disease. Thorax 2008; 63(1):78-84. 